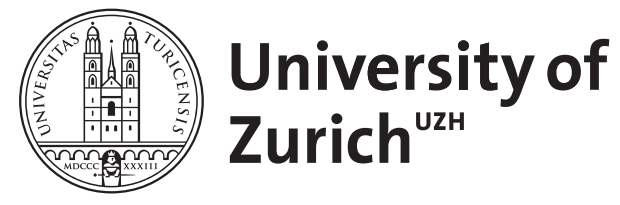
Archive

University of Zurich

University Library

Strickhofstrasse 39

CH-8057 Zurich

www.zora.uzh.ch

Year: 1985

Computer-unterstützte dynamische Thermographie in der Mammadiagnostik

Geser, M ; Bösiger, P

DOI: https://doi.org/10.1515/bmte.1985.30.s1.211

Posted at the Zurich Open Repository and Archive, University of Zurich

ZORA URL: https://doi.org/10.5167/uzh-154339

Journal Article

Published Version

Originally published at:

Geser, M; Bösiger, P (1985). Computer-unterstützte dynamische Thermographie in der Mammadiagnostik. Biomedizinische Technik. Biomedical engineering, 30(s1):211-212.

DOI: https://doi.org/10.1515/bmte.1985.30.s1.211 


\title{
Computer-unterstützte dynamische Thermographie in der Mammadiagnostik
}

\author{
Geser, M. (Zürich), Bösiger, P.
}

Institut für Biomedizinische Technik der Universităt und ETH Zurich, Moussonstr. 18, CH.8044 Z0rich

Die konventionellen mammadiagnostischen Untersuchungsverfahren erfassen vorwiegend strukturelle oder morphologische Gewebeveränderungen. Ergänzend und komplementär dazu liefern thermographische Messverfahren funktionelle Informationen über den Wărmehaushalt der Brust, der beim Vorhandensein eines krebstumors oft gestört ist. Neue Erkenntnisse lassen sich aus der thermographischen Untersuchung des dynamischen Temperaturverhaltens der Brustoberfläche gewinnen. Bei diesem Verfahren wird die auf eine forcierte Abkühlung mit einem Luftstrom folgende wiederaufwarmphase des Gewebes durch periodisches Aufnehmen von Wärmebildern festgehalten. Diese Wärmebildsequenzen werden durch den Computer quant1tativ analysiert.

Ein mögliches Einsatzgebiet der dynamischen Telethermographie in Rahmen der Mammadiagnostik stellt die weitere Abklärung klinisch negativer Fälle dar, die auffällige stationàe Brustthermogramme aufweisen. Zu diesem Zweck wurde ein nach speziellen Fragestellungen ausgewähltes Kollektiv von Patientinnen der senologischen Sprechstunden der Universitätsfrauenklinik Basel $(*)$ dynamisch thermographiert. Die dynamischen Messungen erfolgten unter Standardbedingungen $/ 4 /$, zum Teil unmittelbar nach den statischen Aufnahmen. Bei jeder Untersuchung wurde als erstes die stationare Temperaturverteilung abgespeichert, die als Referenz für die Beurteilung des Abkühlverhaltens dient. Die kühlvorrichtung besteht aus zwei an einem schwenkbaren Hebelarm fest montierten Ventilatoren. Die Kühldauer von 75 Sekunden bewirkt eine Abkühlung der Brustoberfläche von im Mittel 2.5 - 3 Grad C. Nach der Abkühlung wird während der folgenden Wiederaufwärmphase eine Sequenz von 20 Frontalthermogrammen in variablen Zeitintervallen zwischen 3 und 7.5 Sekunden aufgenommen und zur späteren Verarbeitung abgespeichert.

Die für die Steuerung der Aufnahme und die Ver.ır. beitung der Thermogramsequenzen eingesetzte illaye umfasst eine Infrarotkamera, ein Mikroprozes $s^{\prime}, L=Y$ :, tem mit verschiedenen Peripheriegeräten, sowie -in Bildspeicher- und Visualisiereinheit. Mit les fur die Aufnahmen verwendeten IR-Kamera (modifiliertet Thermograph Spectrotherm 2000 von UTI) wesder die Temperaturverteilungen im Bereich von 20 bis $.36 \mathrm{Grad}$ $C$ mit einer Auflösung von etwa 0.1 Grad C registriert. Die digitalisierten Temperaturmesswerte werden in die Bildspeichereinheit übertragen. Der Bildspeicherinhalt wird wahlweise farb- oder yrau- tonkodiert auf einem Monitor dargestellt. Er ist dem Prozessor (Floating-Point CPU TMS 99100A von Texas Instruments) uber ein Memory-Mapping-Verfahren zur Datenanalyse direkt zugänglich. Ein Festplattenlaufwerk dient als Speichermedium für Wärmebildsequenzen und Programme. Die Archivierung der Thermogramme erfolgt in digitaler Form auf videoband, die der Programme auf Floppy-Disk. Ein Lichtgriffel gewährleistet die interaktive Markierung von auszuwertenden Gebieten im Thermogramm sowie die Ablaufsteuerung von Programmen. Zu Dokumentationszwecken stehen eine Farb-Sofortbildkamera und eine $\mathbf{S} / \mathbf{W}$-Hardcopy-Einheit zur Verfügung.

Die bei der Aufnahme auftretenden Bewegungsartefakte infolge der Atmung der Patientin bewirken.Verschiebungen der einzelnen Thermogramme einer Sequenz gegeneinander. Um bei der quantitativen Auswertung der Bildfolgen eine hohe Auflösung und Genauigkeit zu erzielen, müssen diese Artefakte vorgängig möglichst vollständig auskorrigiert werden. Die automatische Bewegungskorrektur durch.den Computer basiert auf einem für diese Zwecke angepassten und optimierten sequentiellen Algorithmus, der translatorische Verschiebungen der Thermogramme bezüglich des ersten Bildes der Sequenz ermöglicht $/ 1,3 /$.

Das Grundziel der Auswertung bildet die Beschreibung der Thermodynamik der auffälligen Strukturen (Gefãssmuster, Hot-Spots) durch quantitative Parameter, mit deren Hilfe eine objektive Klassifikation jeder Sequenz durchgeführt werden kann /3/. Für die Extraktion der interessierenden Strukturen werden Einzelthermogramme und Differenzbilder der Abkühlung und Aufwärmung in mehreren Schritten durch den Computer verarbeitet. Als anomaliesensitiv erweisen sich nicht nur die auf den Differenzthermogrammen definierten parameter, sondern auch Grössen, die das globale und lokale Wiederaufwärmverhalten mit der örtlichen Durchblutung und ihrer Regulation in Kelation stellen. Diese Analyse basiert auf einem iny $\leqslant 1$ kalischen Modell, das die Wàrmebilanz auf der ji..e. beoberflăche berulcksichtigt $/ 2,5 /$.

Die Beurteilung einer Sequenz aufgrund der Gesamtheit aller Parameter beruht auf den Ercjebnıs sen einer multivariaten Diskriminanzanalyse. Dieses Verfahren erlaubt die thermographische Klasseneinteilung der Aufnahmen, basierend auf der Berechmung von Wahrscheinlichkeiten für die Zugehörigkeir. der Messungen zur Gruppe der Sequenzen von Patientinnel

\section{Biomedizinische Technik Band $30 \cdot$ Ergänzungsband $\cdot 1985$ September}


mit negativen und positiven Karzinombefunden.

Das dynamisch untersuchte Lernkollektiv bestand aus 160 Patientinnen ohne nachweisbare Karzinome und 50 Frauen mit histologisch gesicherten Karzinomen. Die retrospektive dynamisch-thermographische Klassifikation der klinisch positiven Fälle ergab eine Karzinomerkennungsrate von etwa $75 \%$, was vergleichbar ist mit der entsprechenden Beurteilung der statischen Warmebilder. Von den 35 Frauen ohne nachweisbare Karzinome mit auffälligen stationären Thermogrammen wurden $40 \%$ dynamisch unauffällig, $20 \%$ dynamisch suspekt und 40 \&ynamisch ebenfalls auffällig klassifiziert.

Die kombinierte Anwendung der statischen und dynamischen Thermographieuntersuchungen kann also die thermographisch "falsch-positiven" Fälle um etwa zwei Funftel reduzieren, wenn dynamisch unauffällige Aufnahmen als thermographisch negativ eingestuft werden. Es bleibt allerdings in einer mehrjährigen klinischen Studie abzuklären, inwiefern sich die Risiken für das Auftreten von Karzinomen zwischen den Gruppen dynamisch auffälliger und dynamisch unauffälliger Patientinnen mit statisch suspekten Thermogrammen unterscheiden.

(*) PD Dr. D. Stucki, Dr. C. Landolt Universitätsfrauenklinik des Kantonsspitals Basel, Schweiz

/1/ Barnea D.I., Silverman H.F. A Class of Algorithms for Fast Digital Image Registration IEEE Trans. Comp. 21 : 179-186, 1972

12/ Bösiger $P$. et al. Dymamische Belastungsthermographie der Gingi\%d Thermologie Fachberichte 4 : 19-22, 1982

/3/ Geser $M$. Diss. ETH Zürich, in Bearbeitung

/4/ Scaroni F. Relevanzanalyse diagnostischer Parameter \%ou Computer-Thermogrammen des menschlichen $r$ ispers Diss. ETH Zürich 7385, 1983

/5/ Thermal Characteristics of Tumours Annals of the New York Academy of Sciences vol. 335, 1980 